\title{
Energetic and Magnetosheath Energy Particle Signatures of the Low-Latitude Boundary Layer at \\ Low Altitudes near Noon
}

Prepared by

J. L. ROEDER and L. R. LYONS

Space and Environment Technology Center

Technology Operations

$$
\begin{aligned}
& \text { HQ GRANT } \\
& 1 N-46-C R
\end{aligned}
$$

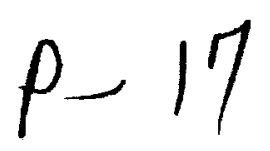

aㅔ

$\begin{array}{lll}+ & & \\ i n & n & 0 \\ i & 0 & m \\ m & 0 & m\end{array}$

15 November 1992

5
0
0
0
0
0

Prepared for

NASA Headquarters

Washington, DC 20546

Grant No. NAGW 1613

Engineering and Technology Group

z山ผ

잉

U느녕

r $-1-2$

(i) $x<0$

$0<1<0$

$x \circ 1$

$\frac{4}{2}>08$

w $5=5$

it

- ZI《0

$\infty$

$\stackrel{0}{M}$ 工

$\rightarrow E C>0$

$\alpha$ \& 4

rum

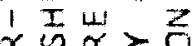

$x \sin 20$

늘룰
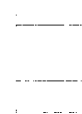
Aerospace Report No.

ATR-91(7190)-2

\title{
ENERGETIC AND MAGNETOSHEATH ENERGY PARTICLE SIGNATURES OF THE LOW-LATITUDE BOUNDARY LAYER AT LOW ALTITUDES NEAR NOON
}

Prepared by

J. L. Roeder and L. R. Lyons Space and Environment Technology Center

Technology Operations

15 November 1992

\author{
Engineering and Technology Group \\ THE AEROSPACE CORPORATION \\ El Segundo, CA 90245-4691
}

Prepared for

NASA Headquarters

Washington, DC 20546

Grant No. NAGW 1613 
Aerospace Report No.

ATR-91(7190)-2

ENERGETIC AND MAGNETOSHEATH ENERGY PARTICLE SIGNATURES OF THE LOW-LATITUDE BOUNDARY LAYER AT LOW ALTITUDES NEAR NOON

Prepared

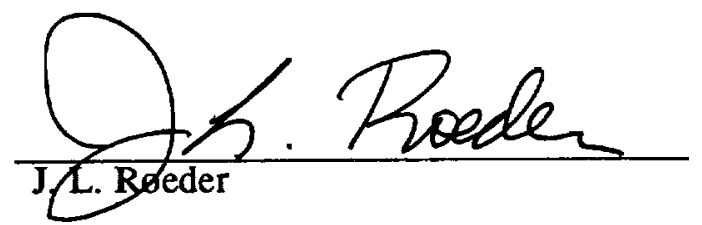

Approved

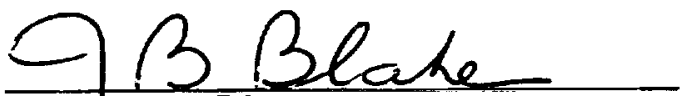

J. B. Blake, Director Space Particles and Fields Department

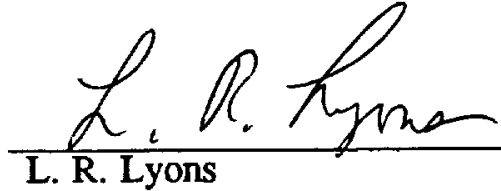

L. R. Lyons

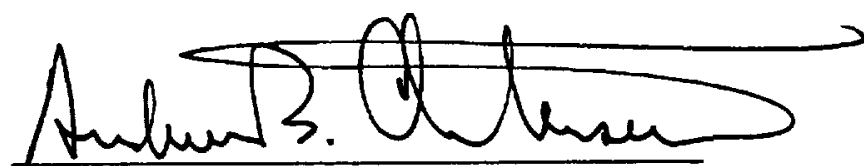

A. B. Christensen, Principal Director Space and Environment Technology Center

ii 


\section{NOTE}

The material reproduced in this report originally appeared in Journal of Geophysical Research. The ATR is published to document the work for the corporate record. 


\title{
Energetic and Magnetosheath Energy Particle Signatures of the Low-Latitude Boundary Layer at Low Altitudes Near Noon
}

\author{
J. L. ROEDER AND L. R. LyONS
}

Space and Environment Technology Center, The Aerospace Corporation, Los Angeles

\begin{abstract}
The low-latitude boundary layer (LBL) and its separation from the cusp have previously been identified using observations of particle precipitation at magnetosheath energies. Using S3-3 satellite observations, we have determined that these identifications can also be made from energetic particle observations on polar-orbiting satellites. It is found that the equatorward boundary of the LBL is identifiable as an approximately discontinuous decrease in $33-\mathrm{keV}$ electron fluxes from low to high latitudes. Both the energetic ion and eiectron fluxes decrease discontinuously at the boundary between the LBL and the cusp or polar cap. A distinct LBL is nearly always identifiable in energetic particle measurements in the 10-14 MLT region when counting rates are statistically significant. The identifications obtained using the energetic particle measurements have been compared to those obtained using criteria developed by Newell and Meng $(1988,1989)$ for magnetosheath enèrgy particle precipitation. In this way, we have evaluated the accuracy of both techniques and used the energetic particle measurements to supplement the identifications obtained using the Newell and Meng criteria. We propose that the Newell and Meng threshold on ion energy flux can be reduced by a factor of 6 . This modification provides identification of the LBL for lower ion intensity levels than has previously been thought possible. Source, acceleration, and scattering processes have also been studied within and in the vicinity of the LBL. Observed trapped pitch angle distributions of energetic electrons imply that the LBL is at least partially on closed field lines. Strong scattering of energetic protons is found within and equatorward of the LBL and thus must occur at least partially along closed field lines. Field-aligned electron acceleration by parallel electric fields can be discerned within and poleward of the LBL, but a more detailed analysis is necessary for a statistical study. Conical ion acceleration was seen relatively frequently within the LBL and about half as often poleward of the LBL. Neither acceleration process could be identified anywhere equatorward of the LBL.
\end{abstract}

\section{INTRODUCTION}

Particles from the Earth's magnetosheath have often been observed to precipitate into the auroral ionosphere over a wide range of local times on the dayside [e.g., Heikkila and Winningham, 1971; McDiarmid et al., 1976; Reiff et al., 1977]. Magnetospheric boundary regions have been associated with this precipitation. Identification of the different boundary layer regions from observations of the magnetosheathlike precipitation would give the ionospheric mapping of the boundary regions and related boundary layer processes. Thus observations of these particles at low altitudes provide a means to monitor the various processes operating to transfer mass, energy, and momentum across the dayside magnetopause (for a review, see Crooker and Burke [1991]).

Newell and Meng [1988, 1989] (hereafter referred to as NM) analyzed precipitating particle data from the lowaltitude, polar-orbiting DMSP spacecraft. They developed rigorous criteria for identifying the cusp and the low-latitude boundary layer (LBL) within the region of magnetosheath particle precipitation. The magnetospheric cusp is characterized by the direct entry of magnetosheath particles along open field lines on the dayside. This results in a fairly high flux of precipitating particles of relatively low average energy. The low-latitude boundary layer is, in contrast, a region that is at least partially on closed field lines and extends into the magnetosphere just inside the magnetopause. It contains a mixture of magnetosheath and magnetospheric particles and produces precipitation with a higher characteristic energy but a lower number flux than occurs

Copyright 1992 by the American Geophysical Union.

Paper number $92 \mathrm{JA00322}$ 0148-0227/92/92JA-00322\$05.00 within the cusp. NM noted that the signature of the LBL at low altitudes has been referred to as the cleft.

In this paper we show that it is also possible to identify the LBL and separate it from the cusp from observations of energetic ( $~ Z 30 \mathrm{keV}$ ) particles at low altitudes. Using S3-3 low-energy and energetic particle data, the LBL identification obtained using the NM criteria is compared with that obtained using energetic particle observations. This comparison allows us to test both identifications. It also shows that the application of both sets of criteria can provide more frequent and reliable identification of the LBL and more accurate identification of its latitudinal extent than can be obtained by using either criterion alone. We also take advantage of the S3-3 particle data to investigate the magnetic field geometry and the occurrence of auroral acceleration processes within the LBL and the cusp.

The NM criteria involve thresholds on the energy flux and average energy of the $32-\mathrm{eV}$ to $30-\mathrm{keV}$ electron and ion precipitation as measured by the SSJ/4 instrument on the DMSP F7 satellite. NM require that the four criteria given in Table 1 be satisfied for a particular region of precipitation to be identified as the LBL. If the average energies of electrons and ions are both below those given by criterion 3 in Table 1 but the other criteria are satisfied, then the region is identified as cusp. If criterion 4 is violated, then the region is identified as plasma sheet (or radiation belts).

The NM studies of dayside DMSP data have recently been extended to identify the plasma mantle and polar rain regions which lie poleward of the cusp and LBL [Newell and Meng, 1990; Newell et al., 1991a]. This report, however, will focus primarily on the signature of the LBL and its separation from the cusp. The simple NM criteria for identification of the regions given have been augmented by a pattern recognition 
TABLE 1. Criteria for Identification as LBL Precipitation

\begin{tabular}{llll}
\hline & Parameter & \multicolumn{1}{c}{ DMSP } & \multicolumn{1}{c}{$\mathrm{S3-3}$} \\
\hline Criterion 1 & $\begin{array}{c}\text { electron energy flux, } \mathrm{eV} \mathrm{s}^{-1} \\
\mathrm{~cm}^{-2} \mathrm{sr}^{-1}\end{array}$ & $J_{e}>6 \times 10^{10}$ & $J_{e}>6 \times 10^{10}$ \\
Criterion 2 & $\begin{array}{l}\text { ion energy flux, } \mathrm{eV} \mathrm{s}^{-1} \mathrm{~cm}^{-2} \\
\mathrm{sr}\end{array}$ & $J_{i}>10^{10}$ & $J_{i}>5 \times 10^{8}$ \\
Criterion 3 & $\begin{array}{l}\text { either electron average } \\
\text { energy, eV } \\
\text { or ion average energy, eV } \\
\text { energy flux of 2- and 5-keV } \\
\text { electrons, eV s-1 } \mathrm{cm}^{-2} \mathrm{sr}^{-1}\end{array}$ & $\begin{array}{l}3000<E_{e}<6000 \\
\text { Criterion 4 }\end{array}$ & $1000<E_{i}$ \\
\hline
\end{tabular}

technique using a neural network algorithm [Newell et al., $1991 b]$. This technique may provide more accurate identification of the various regions, but it provides little quantitative information regarding their physical characteristics. Thus we have used only the NM criteria discussed above.

The data from the S3-3 satellite are particularly advantageous for this study because it was a polar-orbiting satellite at relatively low altitudes that measured energetic particles as well as lower-energy plasma. It was also a spinning satellite, so that particle pitch angle distributions were obtained. The pitch angle distributions of the energetic particles reveal important information on the topology of the local magnetic field lines and on magnetospheric processes. Distributions that are peaked at $90^{\circ}$ and have well-defined minima in both the upgoing and downgoing loss cones are a signature of magnetic trapping on closed field lines. We use this signature of "trapped" distributions to confirm that the LBL lies at least partially on closed field lines. Distributions that are isotropic except for the upgoing loss cone result from strong pitch angle scattering that can be caused by wave-particle interactions or by violation of the guiding center approximation in regions of weak magnetic field. The angular distributions of the lower-energy particles allow us to identify the occurrence of auroral acceleration processes, such as the production of ion conics, within the different regions.

The following section provides a brief review of the relevant instrumentation on the S3-3 spacecraft, a description of several cases of LBL signatures in the S3-3 data, and statistical results using all available S3-3 data from the dayside sector.

\section{OBservations}

The S3-3 spacecraft was launched during July 1976 into an elliptical polar orbit with an initial inclination of $97.5^{\circ}$, an apogee of $8040 \mathrm{~km}$, and a perigee of $240 \mathrm{~km}$ [Cattell, 1982]. The satellite was spin-stabilized in a cartwheel mode with a nominal spin period of $20 \mathrm{~s}$. The instrumentation included two electrostatic analyzer (ESA) sensors measuring electrons from $170 \mathrm{eV}$ to $8.4 \mathrm{keV}$ and ions with energy per charge in the range from $90 \mathrm{eV} / q$ to $3.9 \mathrm{keV} / \mathrm{q}$, each in eight logarithmically spaced channels. A complete energy spectrum was measured by the analyzers once per second. In addition, a magnetic spectrometer measured electrons in the energy range $12-1600 \mathrm{keV}$ in 12 differential energy channels, and a solid-state telescope measured protons in the range $80-1500 \mathrm{keV}$ in five integral energy channels. In this study we use only the channels with the best statistics: the $33-\mathrm{keV}$ and $235-\mathrm{keV}$ channels from the magnetic electron spectrometer and the $>80-\mathrm{keV}$ and $>150-\mathrm{keV}$ channels from the proton telescope.

To select intervals of the S3-3 data for this study, all 86 passes were examined in which the satellite crossed the polar cap (greater than $60^{\circ}$ invariant latitude) at an altitude greater than $1500 \mathrm{~km}$ in the dayside sector (10-14 MLT). The data set was then further restricted to those intervals in which the measured levels of both the energetic electron and ion precipitation were statistically significant (differential electron number flux at $33 \mathrm{keV}$ greater than approximately $10^{2} \mathrm{~s}^{-1} \mathrm{~cm}^{-2} \mathrm{sr}^{-1} \mathrm{keV}^{-1}$ and ion flux at $140 \mathrm{keV}$ greater than $10 \mathrm{~s}^{-1} \mathrm{~cm}^{-2} \mathrm{sr}^{-1} \mathrm{keV}^{-1}$ ). This requirement reduced the data set to 77 satellite passes. Approximately half these cases were selected for this initial study, which resulted in 21 cusp/LBL crossings in which both the energetic particle data and the lower-energy ESA data were available simultaneously for comparison.

\section{Examples}

Plate 1 shows an example of the S3-3 data from March 9, 1977, with the spacecraft in the dayside sector moving equatorward from the polar cap into the radiation belts. Seven and one-half minutes of data are shown as line plots of the energetic particle number fluxes and energy-time spectrograms of the lower-energy plasma number fluxes. The energetic particle fluxes have been averaged over $1-5$ intervals. The top two panels display the electron data, and the bottom two panels show the ion measurements. Note that the top of the electron spectrogram includes the same 33-keV channel as is plotted in the top panel and that the energy scale of the ion spectrogram is inverted. The middle panel shows the pitch angle of the particles sampled as the spacecraft spins, with $0^{\circ}$ designating downgoing particles and $180^{\circ}$ designating upgoing particles for satellite passes in the northern hemisphere (positive latitude). The angles are reversed for southem hemisphere passes (negative latitude). The pronounced spikes in the ion spectrogram once per spin at a pitch angle of approximately $90^{\circ}$ are artifacts due to the response of the ion detectors to solar UV illumination. These sun spikes are well separated from the downgoing loss cone and have little impact on our study of the LBL precipitation.

The spectrograms of Plate 1 show that the particle precipitation prior to $45541 \mathrm{~s}$ UT is observed over the energy range which is typically found in the magnetosheath. NM used similar data to identify the regions of precipitation as the LBL and, further poleward, the cusp. The cusp is clearly identifiable in this example by the characteristic triangular- 


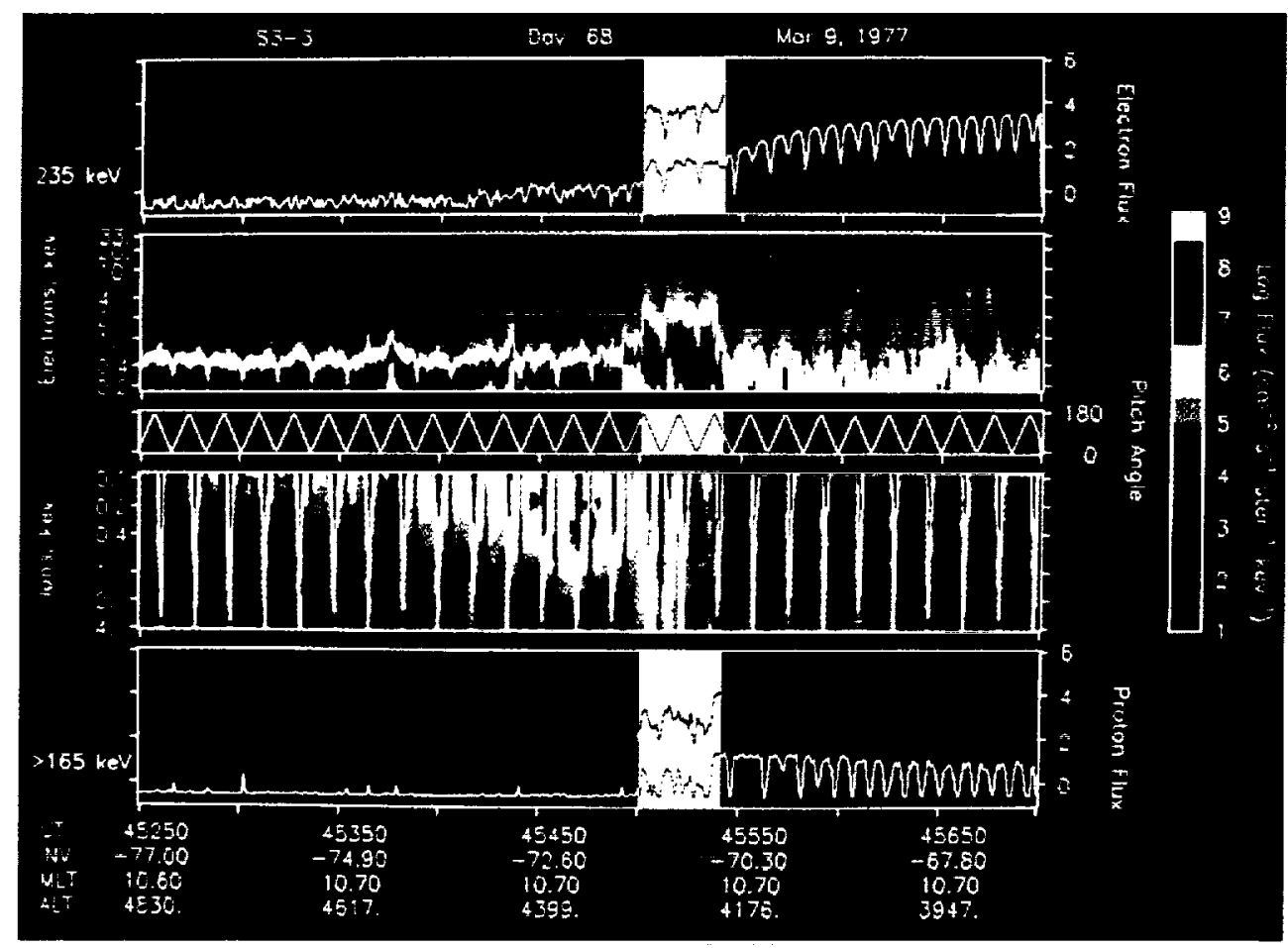

Plate 1. S3-3 particle data during a 7.5-min interval with a crossing of the LBL and cusp. Color energy-time spectrograms display the S3-3 low-energy electron and ion differential number fluxes. The top and bottom panels contain line plots of two channels each of the energetic electron differential number flux and the proton integral number flux. The fluxes from the $>80-\mathrm{keV}$ proton channel have been offset upward by 2 orders of magnitude. The highlighted time interval marks the region identified as the signature of the low-latitude boundary layer in the energetic particle data.

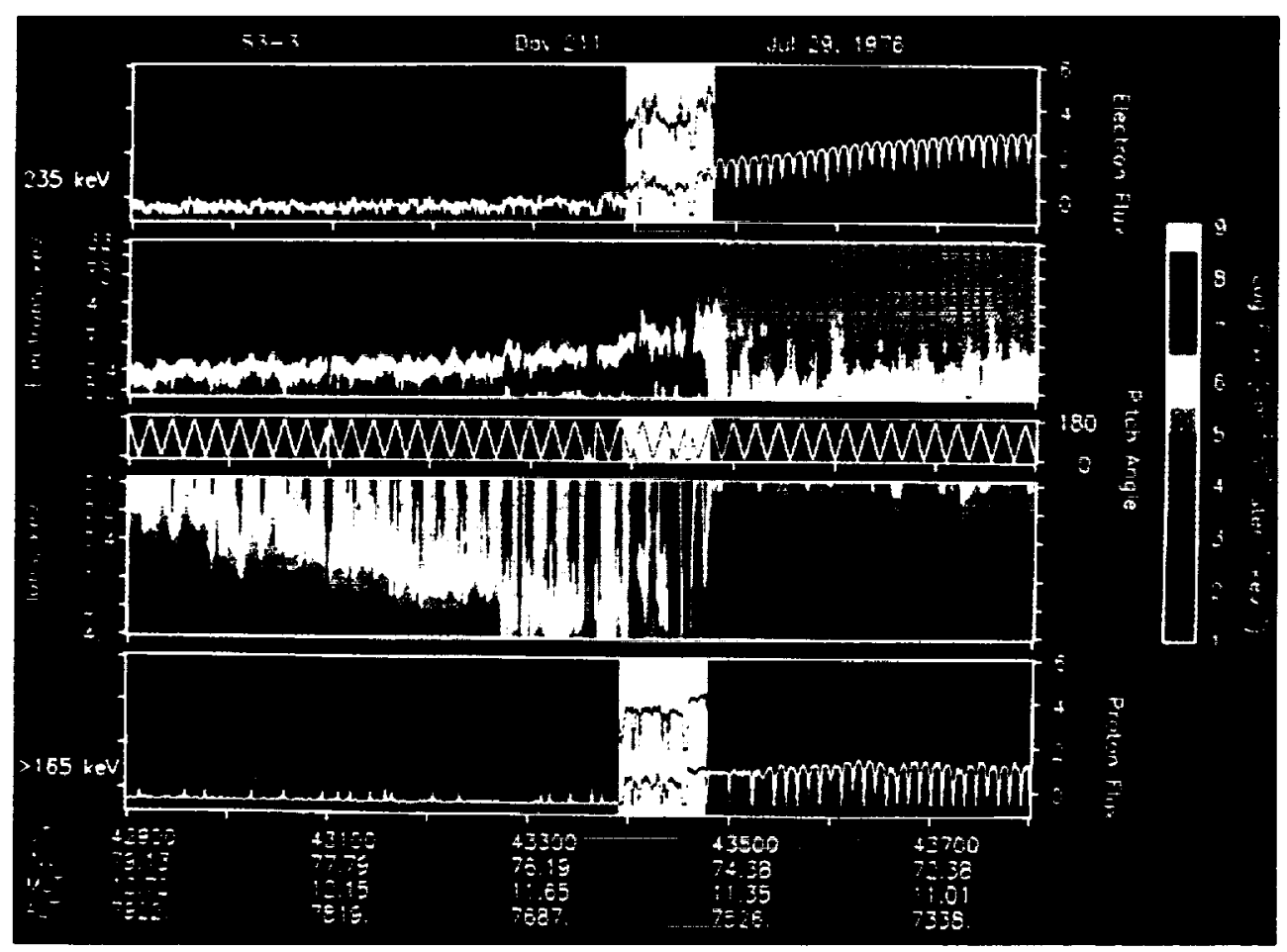

Plate 2. S3-3 low-energy spectrograms and line plots of the energetic fluxes during a second LBL crossing. Conic distributions are visible in the ion spectrogram throughout the LBL and cusp regions. 
shaped region in the ion spectrogram from $-45375 \mathrm{~s}$ to 45500 $s$ UT, where enhanced fluxes extend to increasing energies with decreasing latitude. The energy spectra measured by S3-3 for both the electrons and the ions are reasonably consistent with the other measurements in the magnetosheath and the cusp region [Frank, 1971; Heikkila and Winningham, 1971]. A more detailed comparison between the S3-3 data and other published cusp measurements is reported in the later subsection on statistical results.

The region equatorward of the cusp between $45501 \mathrm{~s}$ and $45541 \mathrm{~s}$ is highlighted in Plate 1 to emphasize the behavior of the energetic electrons. The $33-\mathrm{keV}$ electron fluxes in this region are intermediate in magnitude between the low background of the polar cap on the left side of the figure and the more intense radiation belt fluxes on the right. This interval appears to be a distinct region of the magnetosheathlike precipitation that is distinct from the cusp at higher latitudes and the radiation belts at lower latitudes. Such a feature is typical of all the S3-3 energetic electron data (having statistically significant fluxes) within the dayside sector. The region is often clear in the $235-\mathrm{keV}$ electron channel as well as the 33-keV channel, and it can be identified in the $235-\mathrm{keV}$ data in Plate 1. The edges of this region are sharp and correspond well with discontinuities in the lower-energy electron and ion precipitation in the spectrograms. In particular, the poleward edge of the region is collocated with the equatorward edge of the cusp as identified by the ions. The above characteristics suggest that the energetic particle data can be used as an alternative method to identify the source regions of the precipitation. We propose that the highlighted region be identified as the low-latitude boundary layer, its poleward boundary being at the equatorward boundary of the cusp and its equatorward boundary separating the LBL from the plasma sheet or radiation belts.

The energetic ion data in the bottom panel of Plate 1 show a poleward edge, where fluxes drop to the background levels of the polar cap, that coincides with the poleward edge of the observed energetic electron fluxes. This feature is typical of the data; thus the poleward boundary of the ions can also be used to identify the boundary between the LBL and the cusp. The poleward boundary of energetic ions and electrons is often referred to as the trapping boundary, and it is thought to occur at the boundary between open and closed magnetic field lines. In Plate 1 the energetic ion fluxes are reduced within the highlighted region, as are the energetic electron fluxes. This feature is not commonly found in the S3-3 data. The energetic ion fluxes typically do not show a significant change at the equatorward boundary of the region, so that only the energetic electrons can be used to identify the equatorward boundary of the LBL.

This identification of the LBL based on energetic particle fluxes is consistent with the limited observations of energetic particles within the LBL that have been reported from high altitudes near the magnetopause [Williams et al., 1985; Croley et al., 1986; Song et al., 1990]. These high-altitude observations show that energetic electrons, but not energetic ions, have significantly lower fluxes within the LBL than on lower-latitude field lines.

To test the identification of the LBL using the energetic electron data, we compare it to the identification obtained using the NM criteria on the lower-energy particle precipitation. The differences between the DMSP and S3-3 data, however, require some changes in the criteria in order to make them applicable to the $\$ 3-3$ data. Table 1 shows a comparison of the threshold values for the study of DMSP data by NM and our modified thresholds for the S3-3 data. Most of the changes are due to the more limited energy range of the S3-3 low-energy plasma instrument compared with the DMSP sensors. The NM criteria are based on thresholds on the average energy and total energy flux integrated over the ranges of energy measured by the DMSP sensors. Any similar criteria for the S3-3 data consequently need to be adjusted to the 53-3 energy ranges as closely as possible.

Criterion 3 of the electron and ion average energies listed in Table 1 was readily adjusted to the S3-3 data. The electron data on S3-3 have a lower energy limit of $170 \mathrm{eV}$, whereas the lower limit of the DMSP data is $32 \mathrm{eV}$. The average electron energy within the LBL as measured by the S3-3 detector is thus higher than as measured by the DMSP detector. A wide gap in energy exists between the two S3-3 ion instruments, so that it is impossible to extend the upper limit of the low-energy ion data beyond $3.9 \mathrm{keV}$. This limit of the $\$ 3.3$ ion data, as compared with the DMSP data, has led us to reduce the lower threshold on the average ion energy to $1000 \mathrm{eV}$ and to eliminate the upper threshold.

The major difference between the two sets of criteria is a substantial decrease of the threshold on ion energy flux to 5 $\times 10^{8} \mathrm{eV} \mathrm{s}^{-1} \mathrm{~cm}^{-2} \mathrm{sr}^{-1}$. A factor of 3.3 of this reduction (to a value of $3 \times 10^{9} \mathrm{eV} \mathrm{s}^{-1} \mathrm{~cm}^{-2} \mathrm{sr}^{-1}$ ) is a result of the more limited energy range of the S3-3 ion measurements compared with the DMSP data. A substantial portion of the energy fluxes in the NM data are energies $>3.9 \mathrm{keV}$, and we will show that this reduction in the threshold gives LBL identification results that are consistent with those obtained by NM. However, by using the additional information provided by the energetic particle measurements we have been able to further reduce the ion energy flux threshold by a factor of 6 to the value of $5 \times 10^{8} \mathrm{eV} \mathrm{s}^{-1} \mathrm{~cm}^{-2} \mathrm{sr}^{-1}$. This modification of the NM criteria provides identification of the LBL for lower intensities of ion precipitation than was previously possible.

Figure 1 shows the average energies (in units of $\mathrm{eV}$ ) and total energy fluxes (in units of eV s $\mathrm{cm}^{-1} \mathrm{~cm}^{-1}$ ) computed from the data in the spectrograms of Plate 1. The top two panels give the electron data, and the bottom two panels give the ion data. The full pitch angle variations of the parameters are shown, the satellite spin being most noticeable via the energy flux minima in the upgoing loss cones for ions prior to $45550 \mathrm{~s}$ and for electrons after $45550 \mathrm{~s}$. Note that the sun spikes have been removed from the ion data. The horizontal dotted lines in each panel of Figure 1 show the thresholds in each quantity that are given in Table 1 for identification of the LBL in the S3-3 data. For a region to be identified as the LBL, both the electron and ion energy fluxes must be greater than their respective thresholds. If these conditions are met and the electron average energies are in the range 300-1000 $\mathrm{eV}$, or the ion average energies are greater than $1000 \mathrm{eV}$, then the region is designated to be LBL (unless the electron average energy is $>1000 \mathrm{eV}$ ).

The vertical dotted lines in Figure 1 mark the edges of the LBL region as identified from the energetic electron data in Plate 1. These lines show excellent agreement with the LBL region identified from the criteria of the lower-energy plasma, the two sets of boundaries coinciding to within much less than a satellite spin period in this example. At the equatorward boundary of the LBL the electrons have a 


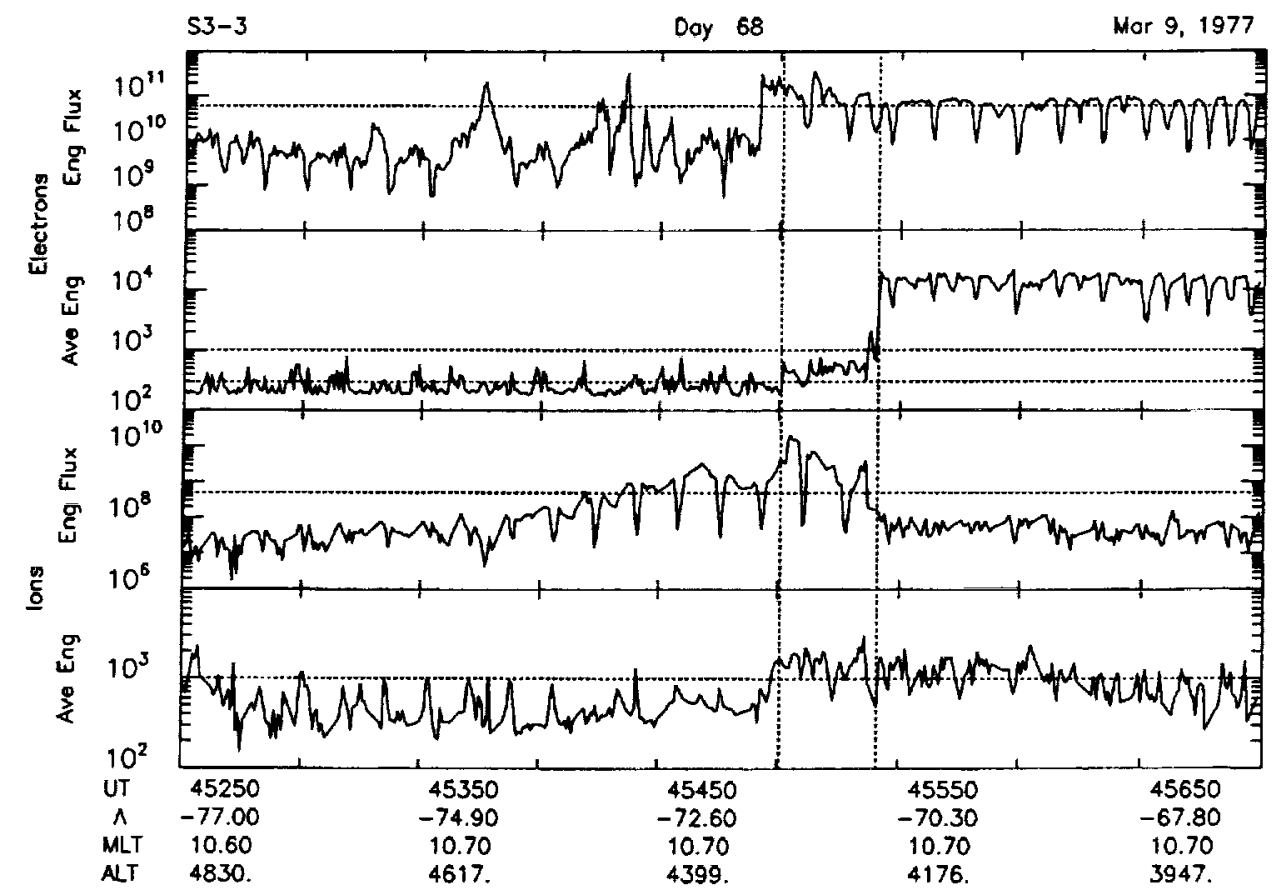

Fig. 1. Total energy flux and average energy computed from the measured 0.170 - to $33-\mathrm{keV}$ electron fux and the 0.09- to 3.9-keV ion flux displayed in Plate 1 . The vertical dotted lines indicate the extent of the low-latitude boundary layer determined from the energetic particle data. The horizontal dotted lines mark the thresholds in each quantity for the criterion given in Table 1 .

dramatic increase in average energy to radiation belt values. This feature is typical of our data. The poleward boundary of the LBL is marked by a decrease in the average electron and ion energies.

A second example from July 29, 1976, is shown in Plate 2 and Figure 2. The cusp in this example is very well defined in the ions and has precipitation extending to energies above $4 \mathrm{keV}$. As in the previous example, the LBL is easily identifiable in the energetic electrons, the poleward boundary being also clearly identifiable in the energetic protons. The equatorward boundary of the LBL is clearly identifiable in the plasma spectrograms in Plate 2 and from the plasma parameters in Figure 2, though the energy flux of downgoing electrons is just barely large enough to satisfy the NM criterion. The poleward boundary of the LBL is identified using the NM criteria by a small change in the electron energy fluxes. The small magnitude of the variations in the low-energy plasma parameters may be due in part to the more limited energy range of the S3-3 instrument compared to the DMSP instrument. These changes by themselves would not give the boundary locations with great confidence. However, the use of both the lower-energy plasma and the energetic particle data provides a clear identification of the boundaries.

The ion spectrogram in Plate 2 clearly shows ions accelerated out of the ionosphere within the cusp and LBL. These upgoing ions, known as ion conics, have enhanced fluxes symmetrically distributed about the flux minima that are centered within the upgoing loss cone [Shelley et al., 1976; Sharp et al., 1977; Mizera and Fennell, 1977; Gorney et al., 1981]. Enhanced fluxes of the conics can be seen extending up to $\sim 0.5 \mathrm{keV}$ within the polar cap after $42900 \mathrm{~s}$. They extend to increasing energies with decreasing latitude, reaching nearly $4 \mathrm{keV}$ within the LBL. No conics are seen equatorward of the LBL. In this particular example, the low-latitude termination of the ion conics can be seen to have been very abrupt and almost precisely at the lowlatitude boundary of the LBL. This is because the equatorward boundary of the LBL occurred almost precisely as $180^{\circ}$ pitch angle particles were being measured, and only one half of the lowest-latitude conical ion distribution was measured.

A third case is shown in Plate 3 and Figure 3, from December 11, 1977. For this example, the LBL is clearly identifiable using the $33-\mathrm{keV}$ electron fluxes as the highlighted region in Plate 3 from $\sim 80805 \mathrm{~s}$ to $80960 \mathrm{~s}$. In this particular case the energetic proton fluxes were too low to obtain statistically significant measurements. From Figure 3 we see that the NM criteria are not satisfied throughout the highlighted region. They are definitively violated for a 40 - to 60 -s interval ( 2 or 3 satellite spins over approximately $1^{\circ}$ in latitude) near $80900 \mathrm{~s}$. Such a major variation is unlikely to be filtered out by any automated algorithm like that of Newell et al. [1991b]. On the other hand, the LBL is clearly identified by the NM criteria equatorward of that region and is marginally identifiable poleward of that region. Despite the fact that the NM criteria are not satisfied throughout the entire LBL region identified by the energetic electrons, the LBL region stands out clearly in both the spectrograms of Plate 3 and the plasma parameters of Figure 3 as an identifiable region that is distinct from the radiation belts and the polar cap. (Energy fluxes were too low poleward of the LBL for the cusp region to be identified with the NM criteria.) This example shows that the energetic particles can be used to identify the entire latitudinal extent of the LBL, when 


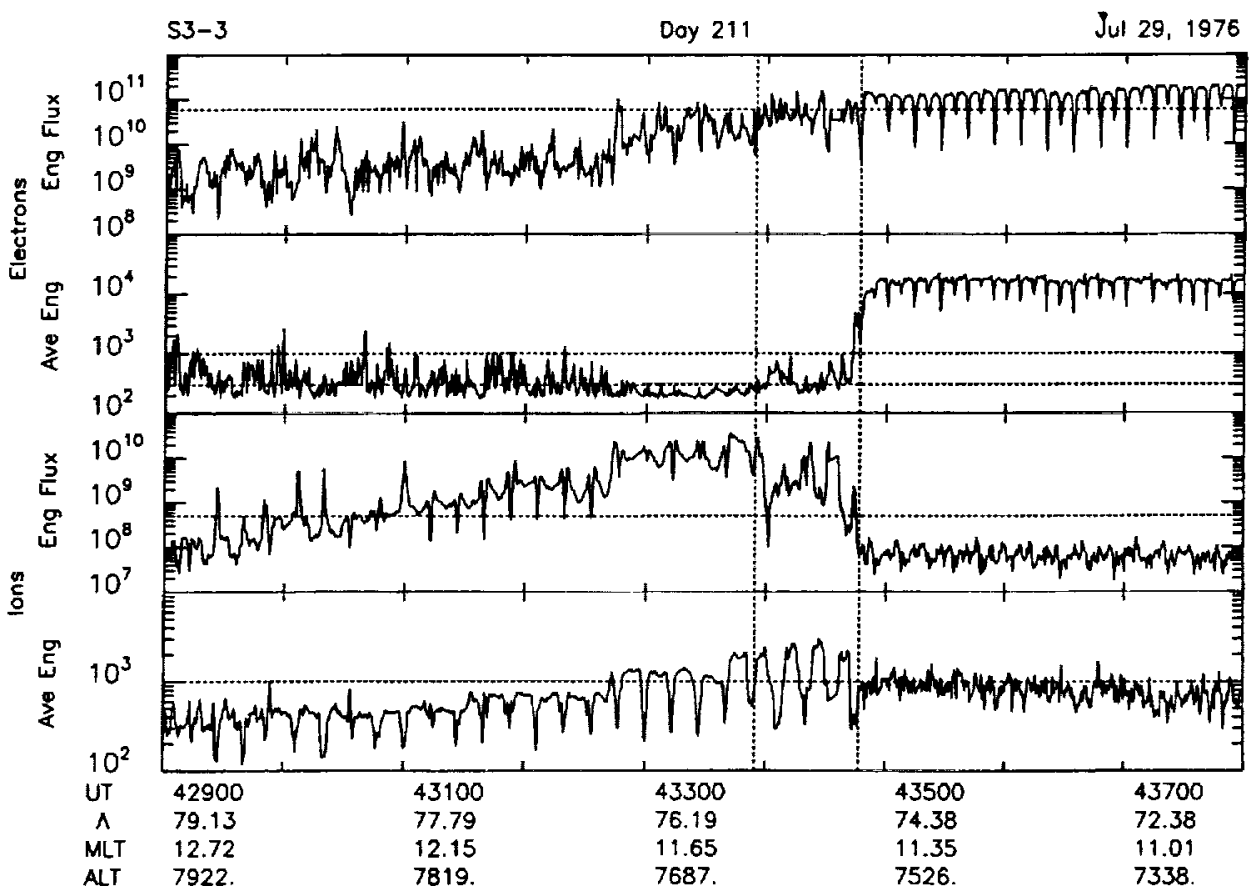

Fig. 2. Total energy flux and average energy computed from the measured low-energy particle fluxes for the interval in Plate 2. The dotted lines again illustrate the agreement between the identification of the LBL using the energetic particles with that resulting from the criteria of the low-energy particle measurements.

only a portion (or portions) of it can be identified using the NM criteria.

A final example is from February 11, 1977. The 33-keV electron fluxes shown in Plate 4 clearly identify a LBL within the highlighted region. However, as can be seen in Figure 4, the NM criteria for LBL identification are not satisfied anywhere within the region. Despite this violation the LBL region is clearly identifiable by the average electron energies in Figure 4, and it shows in the electron spectrogram of Plate 4 as an identifiable region between the polar cap and the radiation belts. This demonstrates that, as should be expected, the rigorous numerical NM criteria can fail to identify the existence of an LBL when one is present, particularly when the magnetosheath energy particle fluxes within the LBL are low. Such examples are relatively rare in our data set, being seen in only two of the 21 orbits. While rare, these examples dramatically demonstrate the value of using energetic particle data to supplement the LBL identifications obtained using the NM criteria of the lower-energy particle data.

"Inverted V" signatures of auroral electron acceleration by parallel electric fields are clearly observable in our spectrograms if the total field-aligned potential drop $\Phi_{\|}$is $\geq 1$ $\mathrm{kV}$. Such occurrences are somewhat rare within the data examined and are not observed in any of the examples presented in this paper. Weaker acceleration regions can more often be discerned (e.g., near $45375 \mathrm{~s}$ UT in Plate 1 and $42350-42375$ s UT in Plate 4), but not with sufficient confidence to study using just the spectrograms.

We have also used the energetic electron measurements to obtain information on the topology of the local magnetic field lines. In the four examples we have shown, the $33-\mathrm{keV}$ and
$235-\mathrm{keV}$ electrons show minima at both $0^{\circ}$ and $180^{\circ}$ pitch angle as well as peaks at $90^{\circ}$ equatorward of the LBL (except for the $33-\mathrm{keV}$ electrons for the two most poleward satellite spins in Plate 1, where pitch angle diffusion that presumably results from wave turbulence caused pitch angle isotropy to extend up into the 33-keV electron channel). These distributions are a well-established feature of radiation belt electrons trapped on closed field lines. The shape of the energetic electron pitch angle distributions within the LBL is less clearly defined by the measurements than it is within the radiation belt because the fluxes are more structured and have worse count rate statistics. However, trapped distributions within the LBL are identifiable at $33 \mathrm{keV}$ and $235 \mathrm{keV}$ for the most equatorward satellite spin in Plate 2, for several satellite spins in Plate 3, and throughout the LBL in Plate 4. These trapped distributions, with clearly defined minima within the downgoing loss cones, imply that at least the equatorward part of the LBL must lie on closed field lines. This is consistent with high-altitude observations that have been interpreted as implying that the $\mathrm{LBL}$ is on closed field lines [Eastman et al., 1976; Eastman and Hones, 1979; Williams et al., 1985].

The angular distributions of the energetic ions also provide useful information regarding processes near the dayside magnetopause. Isotropic energetic ion precipitation has been nearly always observed at all local time [Lundblad et al., 1979]. The isotropic distributions were observed to extend to the poleward edge of measurable fluxes. This isotropic precipitation is seen in the S3-3 energetic proton data when count rates are sufficiently high [Lyons et al., 1987, 1988], and it can be seen in Plates 1 and 2 . It has been suggested that these isotropic distributions result from violation of the 


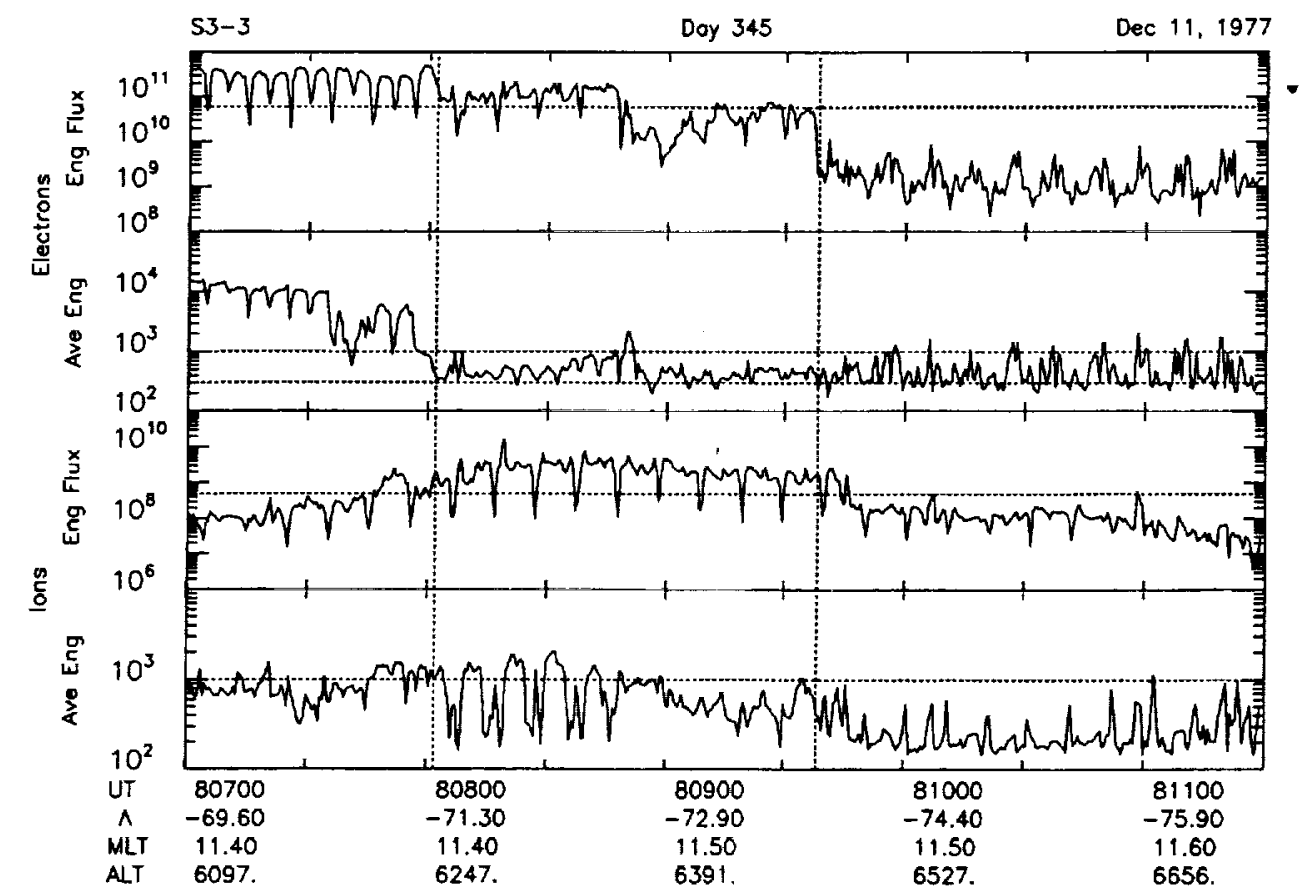

Fig. 3. Total energy flux and average energy computed from the measured low-energy particle fluxes for the interval in Plate 3. The NM criterion identifies the marked interval as several separate regions of LBL in contrast to the single region identified by the energetic electrons.

first adiabatic invariant along magnetic field lines that cross magnetospheric current sheets [Lyons and Speiser, 1982; Lyons et al., 1987]. Such field lines may be closed, as is the case for field lines that traverse the cross-tail current sheet, or they may be open, as is the case for field lines that traverse the magnetopause current sheet.

Lyons et al. [1987] proposed that the nightside precipitation results from scattering in the cross-tail current sheet and that the dayside precipitation may result from previously trapped ions that drift across the boundary between open and closed magnetic field lines and encounter the magnetopause current sheet. If the above suggestions concerning the dayside precipitation were correct, we would expect the equatorward boundary of the isotropic energetic ion precipitation to lie at the boundary between open and closed field lines, coincident with the equatorward boundary of the cusp. We would further expect the LBL to lie equatorward of this boundary if it is entirely on closed field lines.

A quick inspection of Plates 1 and 2 shows that this suggestion is incorrect. Isotropic energetic proton precipitation extends equatorward of the LBL and into regions where the 235-keV electrons exhibit trapped pitch angle distributions. Thus the isotropic ion precipitation extends onto closed magnetic field lines, contrary to the proposal of Lyons et al. [1987]. This suggests that the guiding center approximation is violated for energetic ions on high-latitude, closed field lines on the dayside as well as on the nightside. Low magnetic field magnitudes in the vicinity of the cusps may be responsible for this violation on the dayside (M. Schulz, personal communication, 1991).

\section{Statistical Results}

A survey was performed of the 21 cases in the $1000-1400$ MLT sector in which the energetic electron flux was above the background level. The equatorward edge of the LBL could be discerned from the energetic electron flux in 19 (90\%) of these intervals. This transition always took the form of a discontinuous change in flux to a level between that of the more intense radiation belt fluxes and the background levels of the polar cap. The edge was usually, but not always, better defined in the $33-\mathrm{keV}$ channel than in the $235-\mathrm{keV}$ channel because of its better statistics. The energetic ion precipitation only rarely showed a change which was clearly associated with the equatorward edge of the LBL.

The energetic electron fluxes sometimes decreased with increasing invariant latitude within the LBL sufficiently that count rates became too low to identify the poleward edge. By using only these data, the poleward edge was identified in 15 of the 19 cases with an unambiguous equatorward edge. However, energetic proton fluxes can be sufficiently high for the poleward edge to be identified even when the energetic electron fluxes become too low. By utilizing a combination of the energetic electron and proton fiux data we were able to increase the identification rate to 18 of the 19 intervals. In the remaining case a clear poleward edge of the LBL was not identifiable using the energetic particles, though an approximate location of the edge could be determined. In two (10\%) of the total 21 passes, a direct transition from the plasma 


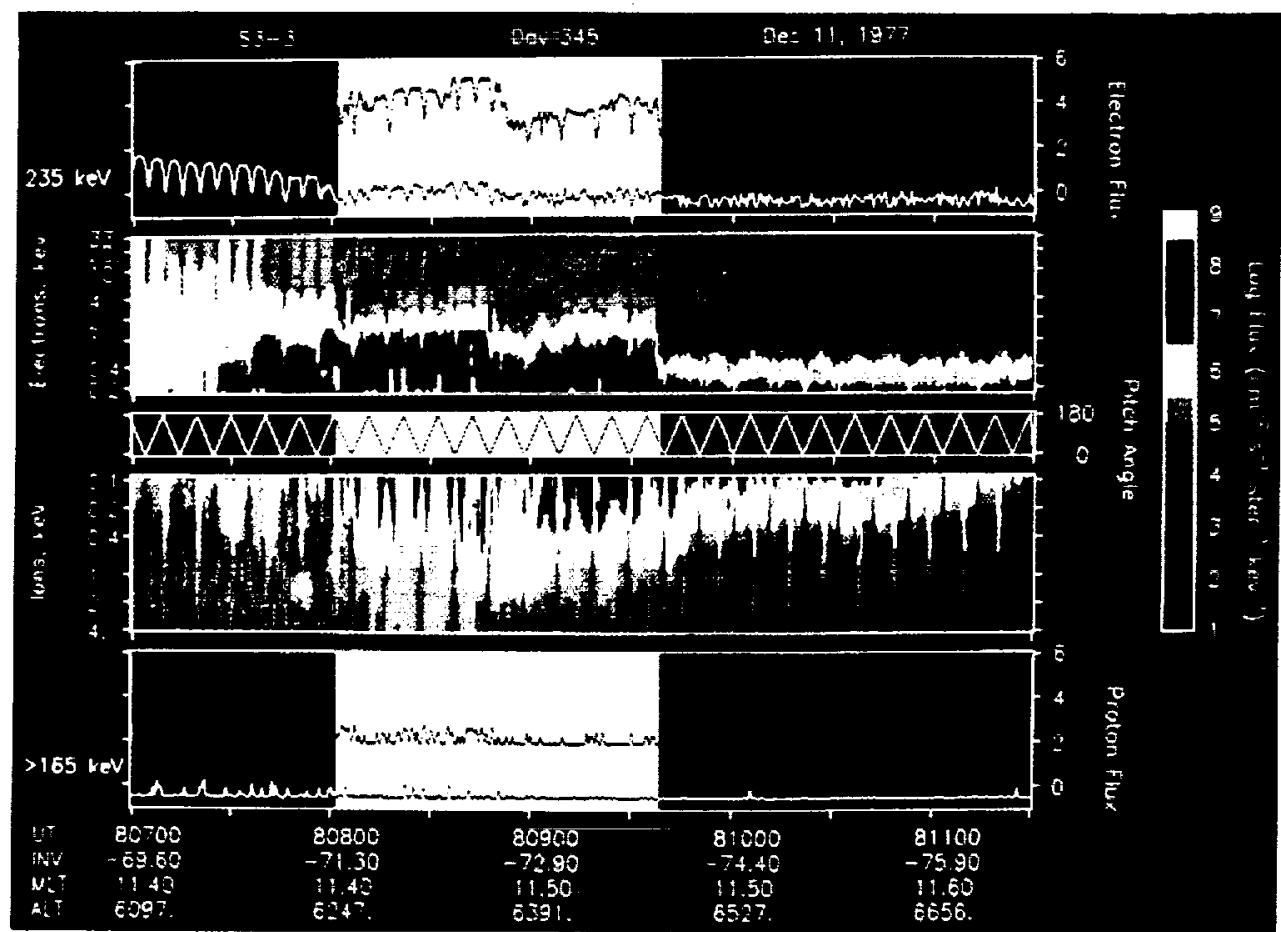

Plate 3. Energy-time spectrograms of the S3-3 low-energy particle fluxes and line plots of the energetic fluxes for a third crossing of the LBL. The energetic particles show a clear signature that can be used to identify the entire latitudinal extent of the LBL.

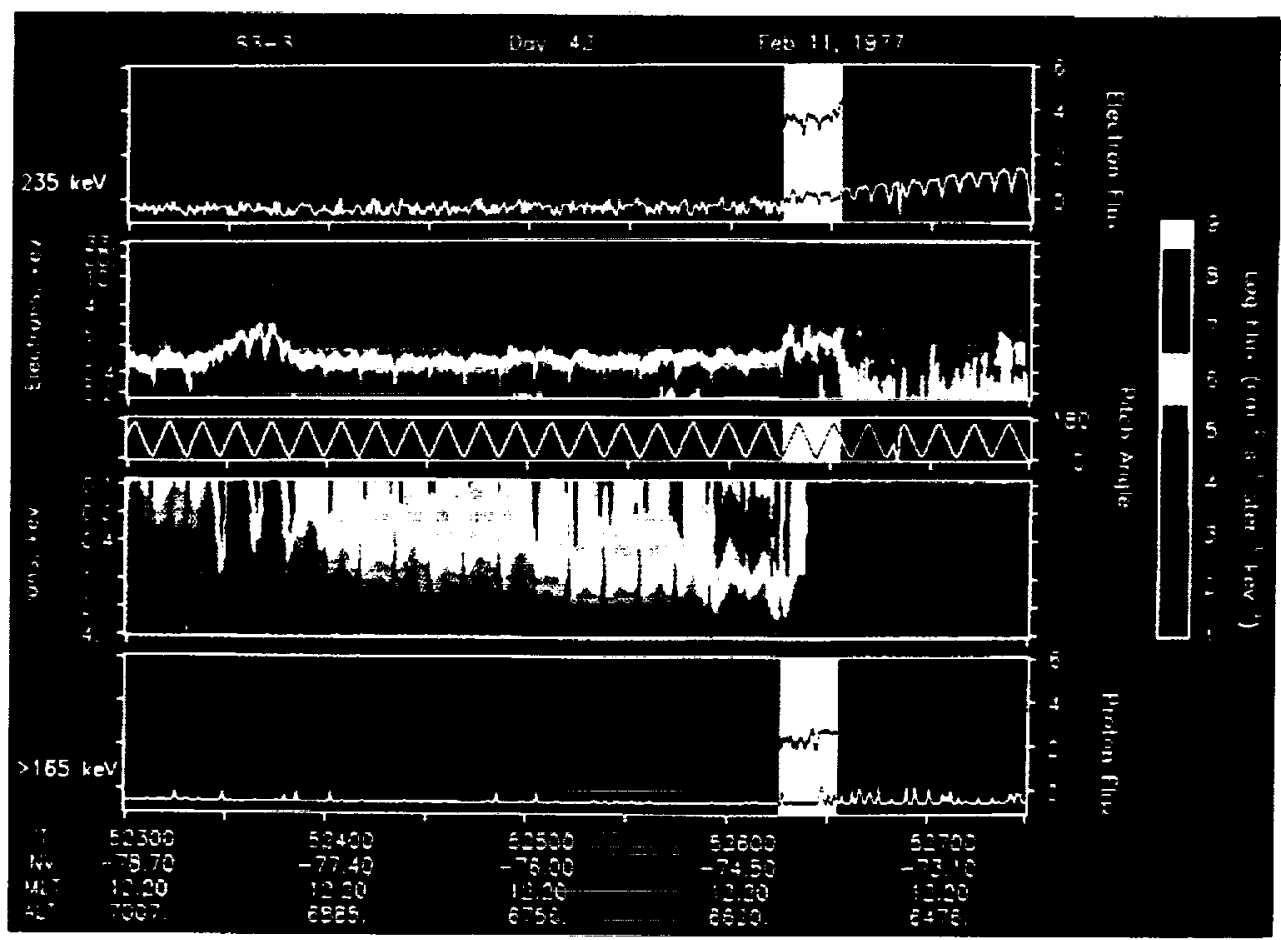

Plate 4. Energy-time spectrograms of the S3-3 low-energy fluxes and line plots of the energetic particles during a fourth LBL crossing. 


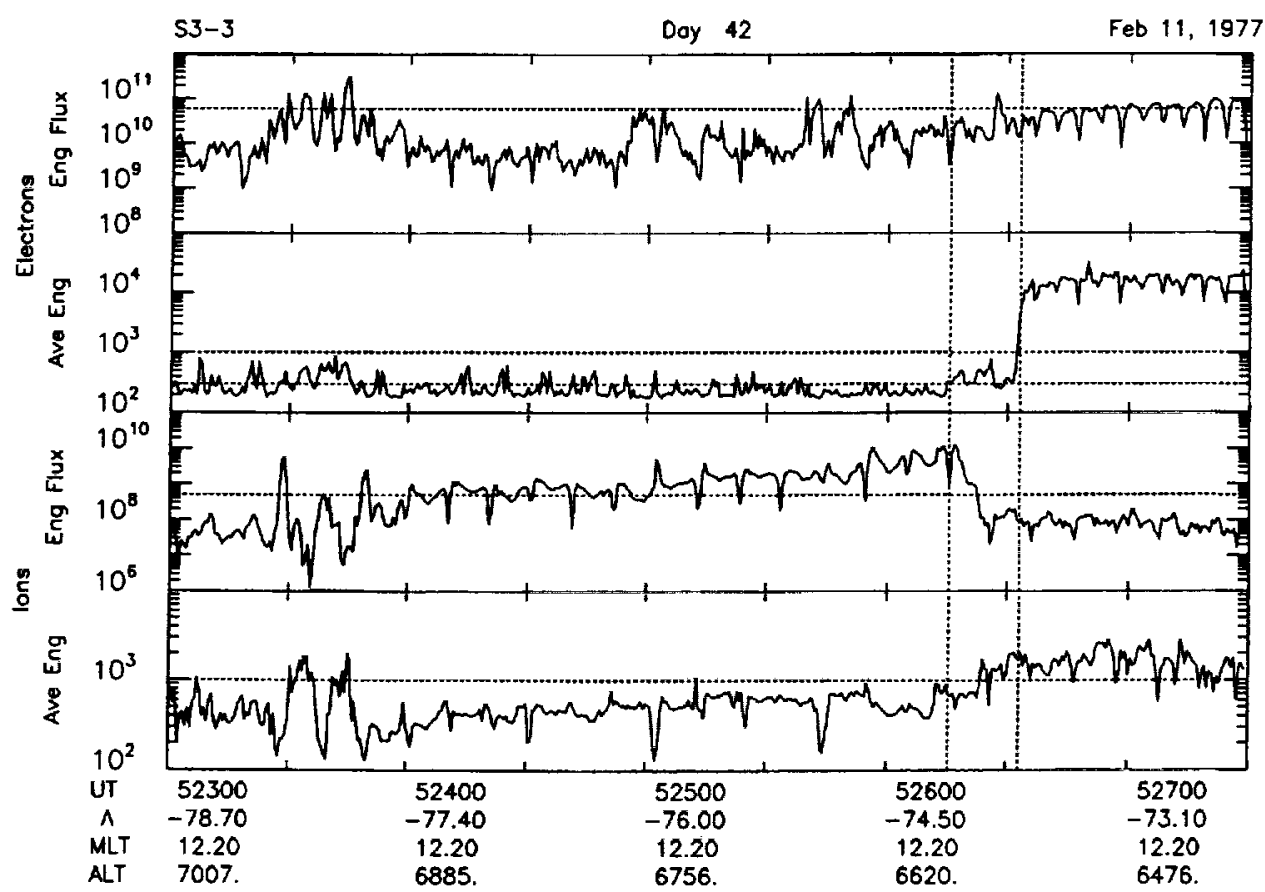

Fig. 4. Total energy flux and average energy computed from the measured low-energy particle fluxes for the interval in Plate 4. This case shows how the criteria of the low-energy particles may fail to identify the LBL when the magnetosheath precipitation is extremely tenuous.

sheet to the cusp was observed without an identifiable LBL region.

We have compared the LBL identifications obtained using the energetic particles to those obtained using our modified NM criteria of the low-energy particles. However, before discussing this comparison, we compare the flux levels observed by S3-3 within the cusp with those that have been reported from other satellites. We do this because the NM criteria use thresholds on absolute fluxes and the comparison can thus be affected by differences in the instrument calibrations.

The energy spectra measured by S3-3 for the electrons are reasonably consistent with the other measurements in the magnetosheath and the cusp. However, for most of the S3-3 orbits, the ion energy spectrum in the cusp exhibited a peak which is below the levels reported by Frank [1971] and Heikkila and Winningham [1971], which are often used as standard references. One example displayed by Frank [1971] from IMP 5 and the several spectra from ISIS 1 [Heikkila and Winningham, 1971] had peaks in the range $4 \times 10^{4}$ to $10^{5} \mathrm{~s}^{-1} \mathrm{~cm}^{-2} \mathrm{sr}^{-1} \mathrm{eV}^{-1}$. S3-3 observed peak fluxes in the range $10^{3}$ to $2 \times 10^{5}$, with $60 \%$ of the 21 cases being a factor of 3 of $10^{4}$. These values are, however, consistent with the values of $2-3 \times 10^{4}$ seen in a second example in the work by Frank [1971] and the values reported by Haerendel et al. [1978]. They are also consistent with the fluxes (peaks of $5 \times$ $10^{3}$ to $10^{4}$ ) measured at low altitudes by AE-C [Potemra et al., 1978; Zanetti et al., 1981]. On the other hand, the peaks are $\sim 10^{5}$ in the DE observations of Burch et al. [1982] and Menietti and Burch [1988].

The above comparisons show that the peak S3-3 fluxes during cusp traversals are as low as $-10^{3} \mathrm{~s}^{-1} \mathrm{~cm}^{-2} \mathrm{sr}^{-1}$
$\mathrm{eV}^{-1}$, whereas other published fluxes generally peak at values above $\sim 10^{4}$. However, the descriptions of the ISIS, $A E, D E$ 1, DE 2, DMSP, and S3-3 instruments reveal that only S3-3 and DMSP had ion detectors sensitive enough to measure fluxes near the $10^{3} \mathrm{~s}^{-1} \mathrm{~cm}^{-2} \mathrm{sr}^{-1} \mathrm{eV}^{-1}$ level. Thus the lack of peak fluxes much below $10^{4}$ on other satellites may well be due to detector sensitivities. The most meaningful comparison for the S3-3 data is with the DMSP data. Plate 6 of Newell and Meng [1989] shows that the yearly average cusp fluxes observed by DMSP peak at $2 \times 10^{4}$. This seems consistent with the S3-3 data, although yearly averages will smear out energy spectral peaks. Of the five individual DMSP passes shown by NM, it should be noted that two cases showed peak fluxes of $2-5 \times 10^{4}$.

We conclude that both the S3-3 ion and electron data are reasonably consistent with the published results. There may, however, be a small discrepancy between the DMSP fluxes and the S3-3 data which is about a factor of $2-3$. This could be a solar cycle variation or another effect, and it should be studied further.

Table 2 summarizes the results of our comparison of the LBL identifications obtained using the energetic particles to those obtained using our modified NM criteria of the lowenergy particles. The quantity listed is the percentage of orbits in which the LBL could be identified by the various methods. The most significant change we have made in the NM criteria is a reduction in the ion energy flux threshold. When we applied the original NM threshold of $10^{10} \mathrm{eV} \mathrm{s}^{-1}$ $\mathrm{cm}^{-2} \mathrm{sr}^{-1}$ to the S3-3 data, we found that a region was identified as LBL in only $29 \%$ of the 21 orbits. This is significantly lower than the occurrence frequency of the LBL in the noon sector reported by NM. We believe that 
TABLE 2. Comparison of Methods for Identification of LBL

\begin{tabular}{ccc}
\hline Criterion & $\begin{array}{c}\text { LBL } \\
\text { Identified, \% } \\
\text { of 21 Orbits }\end{array}$ & $\begin{array}{c}\text { Full Latitude } \\
\text { Range } \\
\text { Identified, } \\
\text { of } 19 \mathrm{LBL} \\
\text { Orbits }\end{array}$ \\
\hline $\begin{array}{c}\text { Energetic particles } \\
\text { NM criteria with ion energy flux } \\
\text { threshold }\end{array}$ & $90 \%$ & $95 \%$ \\
$10^{10} \mathrm{eV} \mathrm{s}^{-1} \mathrm{~cm}^{-2} \mathrm{sr}^{-1}$ & & \\
$3 \times 10^{9} \mathrm{eV} \mathrm{s}^{-1} \mathrm{~cm}^{-2} \mathrm{sr}^{-1}$ & $29 \%$ & $16 \%$ \\
$5 \times 10^{8} \mathrm{eV} \mathrm{s}^{-1} \mathrm{~cm}^{-2} \mathrm{sr}^{-1}$ & $87 \%$ & $37 \%$ \\
\hline
\end{tabular}

this discrepancy is due to the more limited energy range of the S3-3 ion data as compared to the DMSP measurements. Assuming that the spectral shape remains approximately the same, the 53-3 energy range dictates a reduction of the ion energy flux threshold by a factor of 3 . Using a modified threshold value of $3 \times 10^{9} \mathrm{eV} \mathrm{s}^{-1} \mathrm{~cm}^{-2} \mathrm{sr}^{-1}$ resulted in an identification of a region as LBL in $67 \%$ of the cases. This occurrence rate is essentially the same as that obtained by NM in the DMSP study for the noon magnetic local time sector, which indicates consistency between our identification of the LBL in the S3-3 data and the NM identification using the DMSP data. This result gives us confidence that any small calibration differences between the S3-3 and the DMSP sensors do not significantly affect the identification. Also, by using the threshold value of $3 \times 10^{9} \mathrm{eV} \mathrm{s}^{-1} \mathrm{~cm}^{-2}$ $\mathrm{sr}^{-1}$, we never obtained a false identification of the LBL when compared to the identifications obtained from the energetic particle data. All LBL regions identified using the $3 \times 10^{9} \mathrm{eV} \mathrm{s}^{-1} \mathrm{~cm}^{-2} \mathrm{sr}^{-1}$ threshold were entirely within the LBL region identified using the energetic electrons. However, even with this reduced threshold, we missed five of the 19 orbits for which the LBL was clearly identified by the energetic electron data. Also, the full latitude range (to within one satellite spin) of the LBL obtained from the energetic particles was only identified on seven $(37 \%)$ of the orbits. Using the original NM threshold, the full latitude range of the $\mathrm{LBL}$ was only identified on three, or $16 \%$, of the 19 orbits. Note, for example, that the full latitude range of the LBL could not be identified in the case of Plate 2 and Figure 2 using the $3 \times 10^{9} \mathrm{eV} \mathrm{s}^{-1} \mathrm{~cm}^{-2} \mathrm{sr}^{-1}$ threshold.

Comparisons such as in Figure 2 suggest that the threshold on the ion energy flux could be further reduced without yielding false identifications of the LBL. We have found that a reduction to $5 \times 10^{8} \mathrm{eV} \mathrm{s}^{-1} \mathrm{~cm}^{-2} \mathrm{sr}^{-1}$ yields improved agreement between the two techniques for identifying the LBL without providing any false identifications (based on the energetic electron identifications). Using this value for the ion energy flux threshold, a LBL was detected using the low-energy particles in 17 (81\%) of the 21 examined orbits. The LBL was identified in the energetic electrons but not in the low-energy particles in only two (10\%) of the cases, demonstrating excellent agreement between the two techniques.

We also found that the reduced ion flux threshold gave correct identification of the full latitudinal range of the LBL, based on the range obtained from the energetic particles, for $13(68 \%)$ of the 19 LBL orbits. This is approximately twice as often as obtained using the $3 \times 10^{9} \mathrm{eV} \mathrm{s}^{-1} \mathrm{~cm}^{-2} \mathrm{sr}^{-1}$ threshold. In the remaining cases the latitude range deter-
TABLE 3. Location and Occurrence of Particle Phenomena

\begin{tabular}{|c|c|c|c|}
\hline Name & $\begin{array}{c}\text { Equatorward } \\
\text { of LBL }\end{array}$ & $\begin{array}{l}\text { Within } \\
\text { LBL }\end{array}$ & $\begin{array}{l}\text { Poleward } \\
\text { of LBL }\end{array}$ \\
\hline Trapping & $100 \%$ & $69 \%$ & $0 \%$ \\
\hline $\begin{array}{l}\text { Scattering of energetic } \\
\text { protons }\end{array}$ & $74 \%$ & $90 \%$ & $0 \%$ \\
\hline $\begin{array}{l}\text { Electron acceleration by } \\
\qquad \Phi_{\mathrm{y}} \geq 1 \mathrm{kV}\end{array}$ & $0 \%$ & $10 \%$ & $19 \%$ \\
\hline Conical ion distributions & $0 \%$ & $58 \%$ & $29 \%$ \\
\hline
\end{tabular}

Unit is percent of orbits

mined from the low-energy data was fully enclosed by the range identified from the energetic particles. These statistics give further evidence that the reduction of the ion energy flux threshold by a factor of 6 gives improved identification of the LBL, and we feel that this reduction could be applied with confidence to the DMSP data used by NM to allow identification of LBL for lower levels of the ion energy fluxes.

Statistics have also been compiled on phenomena inferred from the angular distributions of the particle fluxes. Table 3 lists the occurrence frequency of several phenomena sorted according to location. We have found that trapped distributions of energetic electrons were observed within the $\mathrm{LBL}$ in $69 \%$ of the 19 cases. Such distributions were observed to extend all the way to the poleward edge of the LBL in four (21\%) of the cases. These results imply that at least part of the LBL generally lies on closed field lines, and they ate consistent with the entire LBL being on closed field lines.

The energetic protons had isotropic angular distributions that were identifiable within the LBL for all orbits (17) for which count rates were sufficiently high for the distributions to be ascertained. These isotropic proton distributions were observed to persist equatorward of the LBL region in 14 (82\%) of the cases. This isotropy implies the existence of a process operating at higher altitudes on closed field lines that scatters particles into the loss cone near the strong diffusion limit.

Identifiable regions of field-aligned electron acceleration by $\Phi \geq 1 \mathrm{kV}$ were relatively rare in our data set. This acceleration was observed twice within the LBL and four times within the region of magnetosheath ion precipitation poleward of the LBL and radiation belts. However, such acceleration often has $\Phi_{\|}<1 \mathrm{keV}$ on the dayside, and a more precise study of such phenomena will require detailed analysis of the electron and ion distributions. The upward acceleration of ions as conical distributions was observed more often, being identifiable within $11(58 \%)$ of the $19 \mathrm{LBL}$ regions and within the region poleward of the LBL and radiation belts on six (29\%) of the 21 orbits. Conical ion distributions were not observed equatorward of the LBL. These observations imply that conical distributions can be formed on both open and closed field lines.

\section{Conclusions}

We have used particle data from the polar-orbiting S3-3 satellite to study features of the low-latitude boundary layer and to study physical processes and particle sources associated with the dayside boundary layers. Previous studies have concentrated on observations of precipitating particles over the range of energies found in the magnetosheath. Here 
we have included the energetic electron and ion data as well as the lower-energy particle data. We have also used angular distributions of the particles. As a result, we have been able to obtain considerably more information than would otherwise be available.

The primary result is that the LBL can be identified from observations of energetic electrons as a region where fluxes are intermediate in magnitude between radiation belt levels and the background levels of the polar cap. When fluxes are sufficiently high that reliable flux measurements can be made, the energetic electron measurements were found to give reliable LBL identification and a definitive determination of its latitudinal extent. Energetic proton fluxes were found to be useful in identifying the poleward edge of the LBL. However, the protons alone were found to be insufficient to identify the presence of the LBL because they do not generally have a detectable signature of its equatorial edge.

We have performed a detailed comparison of the LBL and its boundaries as determined from the energetic particles with the LBL and boundaries determined from a modified set of low-energy plasma criteria based on Newell and Meng [1988, 1989]. We have found excellent agreement in $68 \%$ of the 19 orbits where the LBL was identified by the energetic electron data. In $10 \%$ of the remaining orbits, the modified NM criteria did not provide numerical LBL identification, though a clear boundary region was visually identifiable in the data. For the remaining orbits the modified NM criteria identified the LBL, but not its full latitudinal extent.

Our modified NM criteria differ from the original NM criteria in minor ways (see Table 1) that result from the more limited energy coverage on S3-3 than on DMSP satellites. In addition, a significant reduction has been made in the ion energy flux threshold. A factor of 3.3 of this reduction is due to the more limited ion energy coverage. However, a further factor of 6 reduction has been made in the threshold as a result of the comparisons between the two LBL identification techniques. We propose that this factor of 6 reduction could be reliably applied to NM's DMSP data set without providing false LBL identifications. With this reduction the NM criteria should provide identification of the LBL for lower ion precipitation intensities than was previously thought possible.

We further propose that improved LBL identification can be obtained by simultaneously using both criteria whenever lower-energy and energetic particle data are available from a polar-orbiting satellite. The energetic particle data can provide identification when the lower-energy fluxes are below threshold values, and the lower-energy data can provide identification when the energetic electron count rates are too low for adequate count rate statistics. At times, both techniques can provide identification, thus giving LBL identification with greater reliability than could be obtained from either technique alone.

Our study has also included source, acceleration, and scattering processes operating within and in the vicinity of the LBL. The observed trapped pitch angle distributions of energetic electrons imply that the LBL lies at least partially on closed field lines, in agreement with high-altitude data. The observations are consistent with the entire LBL being on closed field lines and the transition to the cusp occurring at the open-closed field line boundary.

The data also show that isotropic precipitation of energetic ions generally occurs within and equatorward of the lowlatitude boundary layer, and thus it must occur at least partially along closed field lines. This contradicts the suggestion of Lyons et al. [1987] that such precipitation occurs on open field lines that cross the magnetopause current sheet and are adjacent to the open-closed field line boundary. This result implies that the guiding center approximation is violated for energetic protons along closed field lines, a violation that might result from the low magnetic field magnitudes expected near the dayside cusps.

Our examination of acceleration processes suggests that field-aligned electron acceleration by $\Phi_{\|} \geq 1 \mathrm{kV}$ is relatively rare. Conical ion acceleration, on the other hand, was seen relatively frequently within the LBL (on $58 \%$ of the LBL traversals) and about half as often poleward of the LBL. Neither acceleration process could be identified anywhere equatorward of the LBL. Field-aligned electron acceleration by smaller potential drops is probably relatively common, but it cannot be studied in detail using the energy-time spectrograms of the present analysis.

Our initial results have been limited to a study of 21 orbits. It would be desirable to pursue this analysis further using data from additional S3-3 orbits and to repeat the study when data with increased sensitivity become available from a polar-orbiting satellite that measures the angular distribution of lower-energy and energetic particles. For example, we have identified the LBL on $\sim 90 \%$ of dayside orbits by using the energetic particle data. On the other hand, NM identified the LBL on $\sim 70 \%$ of a large number of dayside DMSP orbits, and our identification rate agreed with this when we applied the NM criteria to the S3-3 energy coverage. We found an increase in the identification rate to $\sim 80 \%$ by reducing the ion energy flux threshold. It would be interesting to see if the increase to $\sim 90 \%$ is maintained using energetic particle data from a larger sample of orbits and for electron flux levels which are lower than can be measured with the S3.3 instrumentation.

Acknowledgments. We thank J. F. Fennell and A. L. Vampola for their assistance in analyzing the particle data from S3-3. This work has been suppored by NASA grant NAGW-1613, NSF grant ATM-8800602, and the Aerospace Sponsored Research Program.

The Editor thanks P. T. Newell and another referee for their assistance in evaluating this paper.

\section{REFERENCES}

Burch, J. L., P. H. Reiff, R. A. Heelis, J. D. Winningham, W. B. Hanson, C. Gurgiolo, J. D. Menietti, R. A. Hoffman, and J. N. Barfield, Plasma injection and transport in the mid-altitude polar cusp, Geophys. Res. Lett., 9, 921, 1982.

Cattell, C. A., S3-3 satellite instrumentation and data, in The IMS Source Book, edited by C. T. Russell and D. J. Southwood, p. 91, AGU, Washington, D. C., 1982

Croley, D. R., Jr., J. F. Fennell, and B. G. Ledley, Observation of reconnection phenomena at synchronous orbit, J. Geophys. Res., 9I, 4321, 1986

Crooker, N. U., and W. J. Burke, The cusp/cleft, U.S. Natl. Rep. Int. Union Geod. Geophys. 1987-1990, Rev. Geophys., 29, 1017 , 1991.

Eastman, T. E., and E. W. Hones, Characteristics of the magnetospheric boundary layer and magnetopause layer as observed by IMP 6, J. Geophys. Res., 84, 2019, 1979.

Eastman, T. E., E. W. Hones, Jr., S. J. Bame, and J. R. Asbridge, The magnetospheric boundary layer: Site of plasma, momentum, and energy transfer from the magnetosheath into the magnetosphere, Geophys. Res. Lett, 3, 685, 1976. 
Frank, L. A., Plasma in the Earth's polar magnetosphere, $J$. Geophys. Res., 76, 5202, 1971.

Gomey, D. J., A. Clark, D. Croley, J. Fennell, J. Luhmann, and P. Mizera, The distribution of ion beams and conics below $8000 \mathrm{~km}$, J. Geophys. Res., 86, 83, 1981.

Haerendel, G., G. Paschmann, N. Sckopke, H. Rosenbauer, and P. C. Hedgecock, The frontside boundary layer of the magnetosphere and the problem of reconnection, J. Geophys. Res., 83, $3195,1978$.

Heikkila, W. J., and J. D. Winningham, Penetration of magnetosheath plasma to low altitudes through the dayside magnetospheric cusps, J. Geophys. Res., 76, 883, 1971.

Lundblad, J. A., F. Søraas, and K. Aarsnes, Substorm morphology of $>100 \mathrm{keV}$ protons, Planet. Space Sci., 27, 841, 1979.

Lyons, L. R., and T. W. Speiser, Evidence for current-sheet acceleration in the geomagnetic tail, J. Geophys. Res., 87, 2276, 1982.

Lyons, L. R., A. L. Vampola, and T. W. Speiser, Ion precipitation from the magnetopause current sheet, J. Geophys. Res., 92, 6147, 1987.

Lyons, L. R., J. F. Fennell, and A. L. Vampola, A general association between discrete auroras and ion precipitation from the tail, J. Geophys. Res., 93, 12,932, 1988.

McDiarmid, I. B., J. R. Burrows, and E. E. Budzinski, Particle properties in the dayside cleft, J. Geophys. Res., 8I, 221, 1976.

Menietti, J. D., and J. L. Burch, Spatial extent of the plasma injection region in the cusp-magnetosheath interface, J. Geophys. Res., 93, 105, 1988.

Mizera, P. F., and J. F. Fennell, Signatures of electric fields from high and low altitude particle distributions, Geophys. Res. Lett., 4, 311, 1977.

Newell, P. T., and C.-I. Meng, The cusp and the cleftboundary layer: Low-altitude identification and statistical local time variation, J. Geophys. Res., 93, 14,549, 1988.

Newell, P. T., and C.-I. Meng, On quantifying the distinctions between the cusp and the cleft/LLBL, in Electromagnetic Coupling in the Polar Clefts and Caps, edited by P. E. Sandholt and A. Egeland, pp. 87-101, Kluwer Academic, Boston, Mass., 1989. Newell, $P$. T., and C.-I. Meng, Intense keV energy polar rain, $J$. Geophys. Res., 95, 7869, 1990

Newell, P. T., W. J. Burke, C.-I. Meng, E. R. Sanchez, and M. E.
Greenspan, Identification and observations of the plasma mantle at low altitude, $J$. Geophys. Res., 96, 35, $1991 a$.

Newell, P. T., S. Wing, C.-I. Meng, and V. Sigillito, The auroral oval position, structure, and intensity of precipitation from 1984 onward: An automated on-line data base, J. Geophys. Res., 96, $5877,1991 b$.

Potemra, T. A., J. P. Doering, W. K. Peterson, C. O. Bostrom, R. A. Hoffman, and L. H. Brace, AE-C observations of low-energy particles and ionospheric temperatures in the turbulent polar cusp: Evidence for the Kelvin-Helmholtz instability, J. Geophys. Res. 83, 3877, 1978.

Reiff, P. H., T. W. Hill, and J. L. Burch, Solar wind plasma injection at the dayside magnetospheric cusp, J. Geophys. Res., $82,479,1977$.

Sharp, R. D., R. G. Johnson, and E. G. Shelley, Observation of an ionospheric acceleration mechanism producing energetic (keV) ions primarily normal to the geomagnetic field direction, $J$. Geophys. Res., 82, 3324, 1977.

Shelley, E. G., R. D. Sharp, and R. G. Johnson, Satellite observations of an ionospheric acceleration mechanism, Geophys. Res. Lett., 3, 654, 1976.

Song, P., R. C. Elphic, C. T. Russell, J. T. Gosling, and C. A. Cattell, Structure and properties of the subsolar magnetopause for northward IMF: ISEE observations, J. Geophys. Res., 95, 6375, 1990.

Williams, D. J., D. G. Mitchell, T. E. Eastman, and L. A. Frank, Energetic particle observations in the low-latitude boundary layer, J. Geophys. Res., 90, 5097, 1985.

Zanetti, L. J., T. A. Potemra, J. P. Doering, J. S. Lee, and R. A. Hoffman, Magnetic field-aligned electron distributions in the dayside cusp, J. Geophys. Res., 86, 8957, 1981.

L. R. Lyons and J. L. Roeder, Space and Environment Technology Center, MS: M2-260, The Aerospace Corporation, P. O. Box 92957, Los Angeles, CA 90009.

(Received September 3, 1991; revised February 3, 1992. accepted February 3, 1992.) 


\section{TECHNOLOGY OPERATIONS}

The Aerospace Corporation functions as an "architect-engineer" for national security programs, specializing in advanced military space systems. The Corporation's Technology Operations supports the effective and timely development and operation of national security systems through scientific research and the application of advanced technology. Vital to the success of the Corporation is the technical staff's wide-ranging expertise and its ability to stay abreast of new technological developments and program support issues associated with rapidly evolving space systems. Contributing capabilities are provided by these individual Technology Centers:

Electronics Technology Center: Microelectronics, solid-state device physics, VISI reliability, compound semiconductors, radiation hardening, data storage technologies, infrared detector devices and testing; electro-optics, quantum electronics, solid-state lasers, optical propagation and communications; $c w$ and pulsed chemical laser development, optical resonators, beam control, atmospheric propagation, and laser effects and countermeasures; atomic frequency standards, applied laser spectroscopy, laser chemistry, laser optoelectronics, phase conjugation and coherent imaging, solar cell physics, battery electrochemistry, battery testing and evaluation.

Mechanics and Materials Technology Center: Evaluation and characterization of new materials: metals, alloys, ceramics, polymers and their composites, and new forms of carbon; development and analysis of thin films and deposition techniques; nondestructive evaluation, component failure analysis and reliability; fracture mechanics and stress corrosion; development and evaluation of hardened components; analysis and evaluation of materials at cryogenic and elevated temperatures; launch vehicle and reentry fluid mechanics, heat transfer and flight dynamics; chemical and electric propulsion; spacecraft structural mechanics, spacecraft survivability and vulnerability assessment; contamination, thermal and structural control; high temperature thermomechanics, gas kinetics and radiation; lubrication and surface phenomena.

Space and Environment Technology Center: Magnetospheric, auroral and cosmic ray physics, wave-particle interactions, magnetospheric plasma waves; atmospheric and ionospheric physics, density and composition of the upper atmosphere, remote sensing using atmospheric radiation; solar physics, infrared astronomy, infrared signature analysis; effects of solar activity, magnetic storms and nuclear explosions on the earth's atmosphere, ionosphere and magnetosphere; effects of electromagnetic and particulate radiations on space systems; space instrumentation; propellant chemistry, chemical dynamics, environmental chemistry, trace detection; atmospheric chemical reactions, atmospheric optics, light scattering, state-specific chemical reactions and radiative signatures of missile plumes, and sensor out-of-field-of-view rejection. 